\title{
Leptin Regulated ILC2 Cell through the PI3K/AKT Pathway in Allergic Rhinitis
}

\author{
Qingxiang Zeng $\left(\mathbb{D}, X^{2}\right.$ Luo $\mathbb{D}^{D}$, Yiquan Tang, Wenlong Liu $(\mathbb{D}$, and Renzhong Luo \\ Department of Otolaryngology, Guangzhou Women and Children's Medical Center, Guangzhou Medical University, \\ Guangzhou, China \\ Correspondence should be addressed to Wenlong Liu; lwl20103@163.com and Renzhong Luo; 691285341@qq.com
}

Received 8 November 2019; Revised 7 January 2020; Accepted 11 February 2020; Published 7 March 2020

Academic Editor: Kerstin Wolk

Copyright ( 2020 Qingxiang Zeng et al. This is an open access article distributed under the Creative Commons Attribution License, which permits unrestricted use, distribution, and reproduction in any medium, provided the original work is properly cited.

\begin{abstract}
Background. Recent studies suggest that leptin is involved in Th2 response in allergic rhinitis (AR). However, the effect of leptin on type II innate lymphoid cells (ILC2s) in AR is not well characterized. Methods. Twenty-six AR patients and 20 healthy controls were enrolled. Serum leptin levels were measured, and their correlation with ILC2 and type II cytokines were analyzed using enzymelinked immunosorbent assay (ELISA) and flow cytometry. ILC2 differentiation and cytokine production stimulated by human recombinant leptin were analyzed by real-time polymerase chain reaction (PCR) and ELISA. AR mouse models were also established to verify the effect of leptin on ILC2 cell regulation. Results. Our results showed that elevated serum leptin in AR patients was correlated with the percentage of ILC2 and the expression of type II cytokines. The recombinant leptin enhanced the expression of ILC2 cell transcription factors and type II cytokine through the PI3K/AKT pathway. The AR mice treated with leptin showed as stronger ILC2 inflammation and symptoms compared with control mice. Conclusions. Our data provide evidence that upregulation of leptin promotes ILC2 responses in AR and this process was achieved through the PI3K/AKT pathway.
\end{abstract}

\section{Background}

Epidemiological survey shows that there are about 500 million allergic rhinitis (AR) patients worldwide, affecting the quality of life of $10 \%-20 \%$ of the population [1]. In recent years, the incidence of $\mathrm{AR}$ in China has been increasing year by year. It is worth noting that the incidence of obesity is also increasing synchronously [2]. Obesity has been proved to be an important risk factor for the occurrence and development of respiratory allergic inflammation (especially asthma) [3].

White adipose tissue can mediate metabolic effects by secreting a large number of adipose factors in obese patients, including leptin and adiponectin, in which leptin is considered to be the core medium linking nutrition, metabolism, and immune homeostasis [4]. Leptin has been proved to promote Th1 response in both humans and mice, but its role in Th2 response remains unclear [5-7]. Previous studies have shown that the serum levels of leptin in patients with AR are significantly higher and positively correlated with the severity of clinical symptoms $[8,9]$. The combination of leptin and its receptor activates JAK2-STAT3, MAPK, and PI3K-AKT pathways $[10,11]$.

ILC2 is widely distributed in adipose-associated lymphoid tissue, intestine, lung, and skin and is an important member of the early stage of immune response. Allergens directly activate ILC2 by inducing the secretion of epithelial-derived cytokines such as IL-25, IL-33, and TSLP to produce IL-13, IL-5, IL-4, and IL-9 [12]. Studies had found that ILC2 in lungs of obese mice did not decrease despite that the number of ILC2 in visceral adipocytes of obese patients and mice decreased significantly $[13,14]$. These results suggested that ILC2 was significantly affected under obese state. Studies also showed that the frequency of ILC2s was significantly decreased by high-fat-diet feeding and leptin deficiency-induced obesity [15]. 
In this study, we aimed to explore the effect of human recombinant leptin on the differentiation and function of ILC2 by both in vivo and in vitro studies.

\section{Methods}

2.1. Patients. Twenty-six AR patients without obesity and twenty healthy controls without obesity were recruited in this study. As described in Allergic Rhinitis and its Impact on Asthma guideline (2010), AR was diagnosed according to symptoms and duration, allergens test to common inhalant allergens (dust mites, pets, molds, cockroach, etc.) by skin prick test or specific IgE measurement [1]. The exclusion criteria included the following: atopic dermatitis, asthma, nasal anatomic abnormalities, and use of systemic corticosteroids in the previous 2 months. The study protocols were approved by local ethics committee boards, and written informed consent was obtained.

2.2. Flow Cytometry for ILC2. Peripheral blood mononuclear cells (PBMCs) were prepared using Lymphoprep (Fresenius Kabi Norge AS, Oslo, Norway) density-gradient centrifugation from heparinized leucocyte-enriched buffy coats. Isolated PBMCs were cultured at $2 * 10^{6} / \mathrm{mL}$ in 24 -well plates supplemented with RPMI-1640 with 5\% human AB serum, $5 \mathrm{mmol} / \mathrm{L}$ glutamine, and penicillin and streptomycin solution (all from Invitrogen, except serum from Sigma-Aldrich). Then, PBMCs were stimulated by PMA $(50 \mathrm{ng} / \mathrm{mL})$ and ionomycin $(500 \mathrm{ng} / \mathrm{mL}$; both from Sigma-Aldrich) for 4 hours and by brefeldin A (BD, Oxford, United Kingdom) for the final 3 hours of culture.

PBMCs from donors were stained by antibody mixture (FITC lineage cocktail: CD2(RPA-2,10), CD3(OKT3), CD14(61D3), CD16(CB16), CD19(HIB19), CD56(TULY56), CD235a(HIR2), eBioscience, San Diego, CA) and FceRI (9E1, eBioscience) for the exclusion of $\mathrm{B}, \mathrm{T}$, natural killer, and natural killer $\mathrm{T}$ cells as well as mast cells and basophils. This process left approximately 50\% pure $\mathrm{Lin}^{-}$cells. The Lin cells were then stained with APC/Cy7-conjugated CD45 antibody (2D1), PE-conjugated CRTH2 antibody (BM16, BD Biosciences, NJ), and PE-Cy7-conjugated CD127 antibody (HIL7R-M21, BD Biosciences, NJ) for the identification of human ILC2s. The human ILC2s were identified as $\mathrm{Lin}^{-} \mathrm{CD} 45^{+}$ $\mathrm{CRTH} 2{ }^{+} \mathrm{CD} 127^{+}$lymphocytes. The mice ILC2s in PBMCs were identified as $\operatorname{Lin}^{-}$(TCR $\beta(\mathrm{H} 57,597), \mathrm{TCR} \gamma \delta(\mathrm{eBioGL} 3)$, CD3 $\varepsilon(145,2 \mathrm{C} 11)$, Gr-1(RB6,8C5), CD11b(M1/70), TER119(TER,119), B220(RA3,6B2), NK1.1(PK136), CD5(53,7.3), and $\mathrm{CD} 11 \mathrm{c}(\mathrm{N} 418)) \mathrm{ST}^{+}(\mathrm{RMST} 2,2) \mathrm{CD} 45^{+}(104) \mathrm{CD}^{+} 7^{+}$ (A7R34) lymphocytes. IL-5(JES1-39D10), IL-4(3010.211), and IL-13 (JES10-5A2) positive ILC2 cells in PBMCs were determined by intracellular cytokine staining using Cytofix (BD Biosciences, NJ) according to the manufacturer's instructions. The Beckman flow cytometer machine was used in the test (Beckman Coulter, Hercules, CA, USA).

2.3. ILC2 Sorting. Using the EasySep FITC selection kit, the peripheral blood lineage positive $\left(\mathrm{Lin}^{+}\right)$cells in PBMCs were removed, and then lineage negative $\left(\mathrm{Lin}^{-}\right)$cells were enriched. Then, the Lin $^{-} \mathrm{CRTH} 2^{+} \mathrm{CD} 127^{+}$cells $\left(1.5 \times 10^{5}\right.$
TABLE 1: Demographic characteristic of AR and control patients.

\begin{tabular}{lcc}
\hline Groups & AR group & Control \\
\hline Number & 26 & 20 \\
Sex (male : female) & $14: 12$ & $11: 9$ \\
Age (years) & $29.8(18-51)$ & $32.4(21-56)$ \\
BMI & $20.3(18.9-22.4)$ & $21.6(19.2-23.1)$ \\
ECP (ng/mL) & $61.3(22.6-127.3)^{*}$ & $18.1(7.5-98.2)$ \\
TIgE $(I U / m L)$ & $148.9(37.2-638.2)^{*}$ & $31.5(9.1-56.7)$ \\
\hline
\end{tabular}

${ }^{*}$ Compared with the control group, $P<0.05$.

cells/mL) were enriched through staining with PE-labeled CRTH2 and PE-Cy7-labeled CD127 using a MoFlo XDP cell sorter (Beckman Coulter, CA, USA) and cultured in RPMI-1640 with 10\% FBS and 1\% penicillin/streptomycin for 3 days. The viability was larger than 98\% (Trypan blue staining). At the same time, IL-25 (10 ng/mL), IL-33 (10 ng/mL), TSLP (10 ng/mL), and IL-2 (50 ng/mL) were added. For stimulation experiments, $100 \mathrm{ng} / \mathrm{mL}$ leptin, $100 \mathrm{ng} / \mathrm{mL}$ LY294002 (PI3K specific inhibitor), $100 \mathrm{ng} / \mathrm{mL}$ AG490 (JAK inhibitor), and $100 \mathrm{ng} / \mathrm{mL}$ SB203580 (MAPK inhibitor) were added. PBS was used as negative control. All cytokines and inhibitors were purchased from R\&D Systems. A dose response experiment was performed to determine the concentration of stimulators. The stimulation concentrations of different stimulators were confirmed in pretest. The proliferation rate was assessed through incorporation of tritiated thymidine.

2.4. Quantitative Real-Time PCR ( $q R T-P C R)$. The total RNA was isolated from ILC2s with TRIzol reagent (Life Technologies, Carlsbad, California). RNA (1 $\mu \mathrm{g})$ was digested by DNase I, extracted with phenol:chloroform (3:1), precipitated with ethanol, washed with ethanol, dissolved in RNAse-free water, and reverse-transcribed by the cDNA kit (Qiagen). PCR amplification was detected using an ABI PRISM 7300 Detection System. The data were calculated by the $2^{-\Delta \Delta \mathrm{Ct}}$ method. The relative expression of target genes was normalized to GAPDH. The primers used in the test were listed as follows: GATA3 sense, $5^{\prime}$-GCGGGCTCTAT CACAAAATGA-3', antisense, $5^{\prime}$-GCTCTCCTGGCTGC AGACAGC-3'; ROR $\alpha$ sense, 5'-AAGGAGCCAGAAGG GATGAAC- $3^{\prime}$, antisense, $5^{\prime}$-GGAACA ACAGACGCCAG TAAG-3'; GAPDH sense, 5'-AGCCACATCGCTCAGA CAC- $3^{\prime}$, antisense, $5^{\prime}$-GCCCAATACGACCAAATCC- $3^{\prime}$.

2.5. Western Blot. ILC2 cells were lysed by cold RIPA buffer, and the samples were separated using 10\% SDSpolyacrylamide gel electrophoresis (PAGE). After blocked by $5 \%$ TBST-milk for 60 minutes, PVDF membranes were incubated with mouse anti-pAKT (5.Ser 473) and $\beta$-actin (clone:C4) (Santa Cruz, $1: 1000$ ) overnight under $4^{\circ} \mathrm{C}$. After incubation with horseradish peroxidase-labeled anti-rabbit IgG antibody, the signals were measured by the enhanced chemiluminescence kit (Pierce, Rockford, IL).

2.6. Enzyme-Linked Immunosorbent Assay (ELISA). The concentration of type II cytokines (IL-4, IL-5, and IL-13) in the 

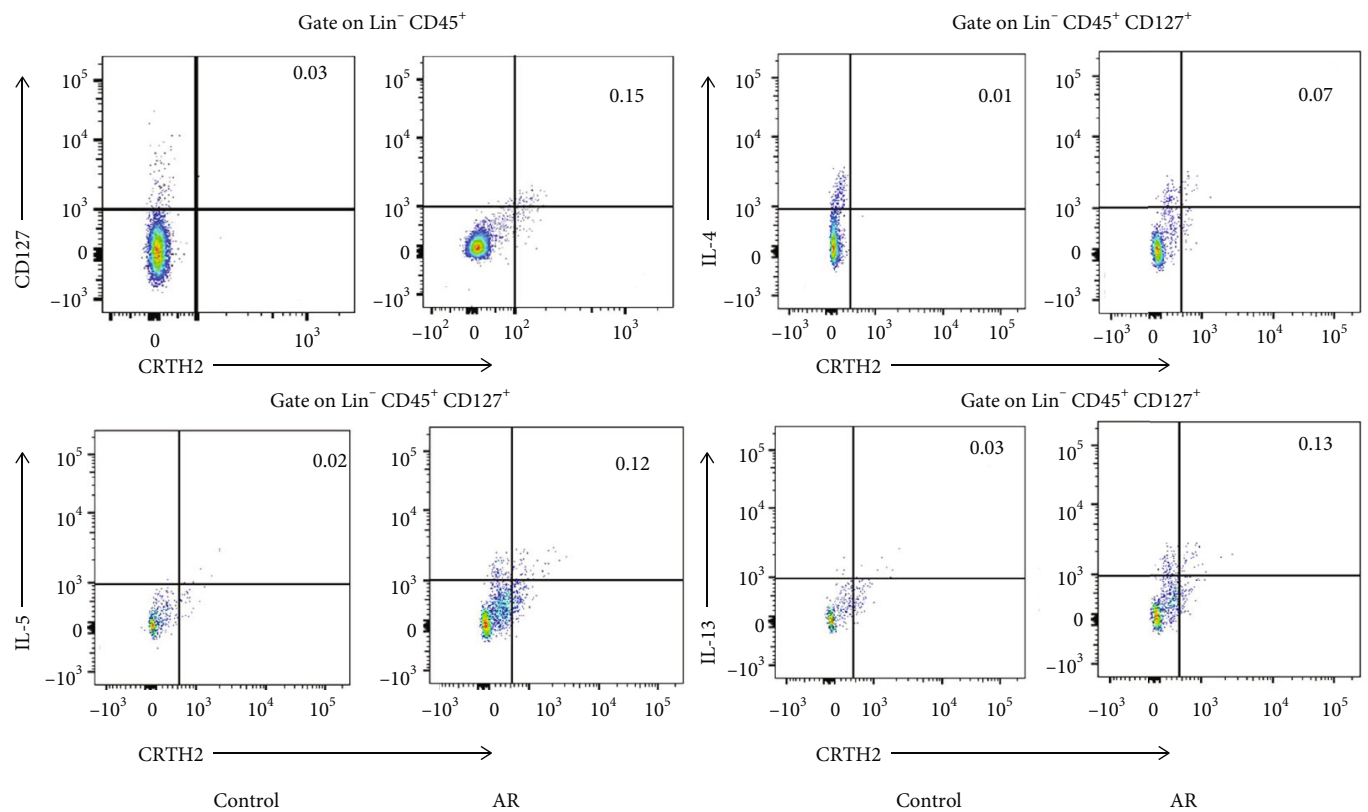

FIGURE 1: Representative flow cytometry showed elevated ILC2 ( $\operatorname{Lin}^{-} \mathrm{CD} 45^{+} \mathrm{CRTH} 2^{+} \mathrm{CD} 127^{+}$) frequency, IL- $4^{+}$ILC2 frequency, IL- $5^{+}$ILC2 frequency, and IL-13 ${ }^{+}$ILC2 frequency in PBMCs from AR compared with controls. All the frequencies of ILC2s were converted into proportions in PBMCs (per $\mathrm{mL}$ of blood). Three independent tests were performed for every experiment.

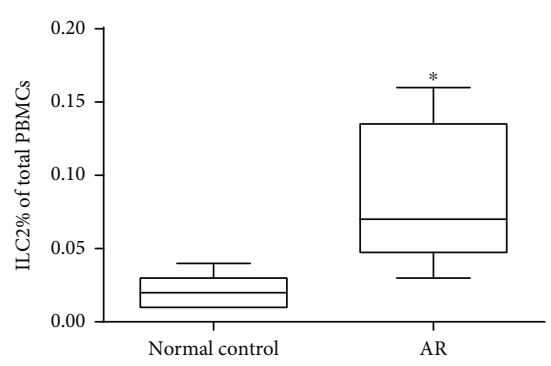

(a)

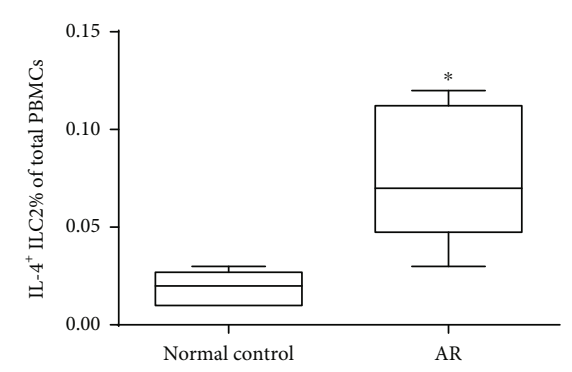

(b)

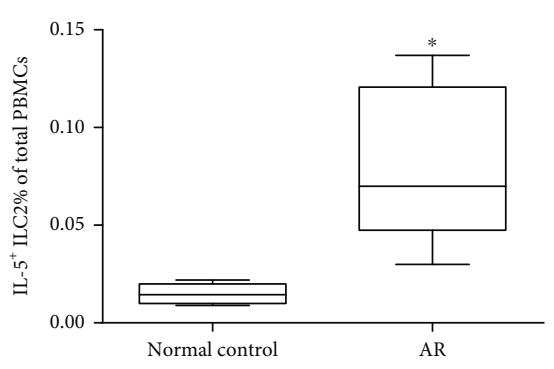

(c)

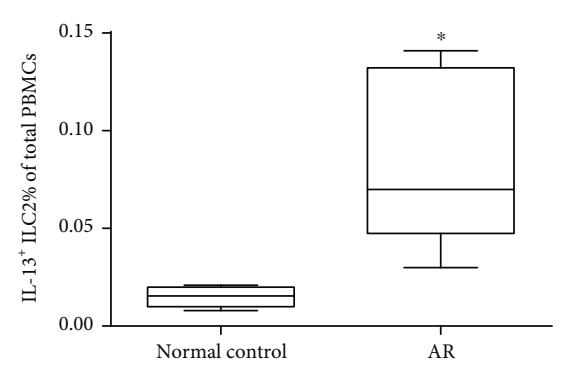

(d)

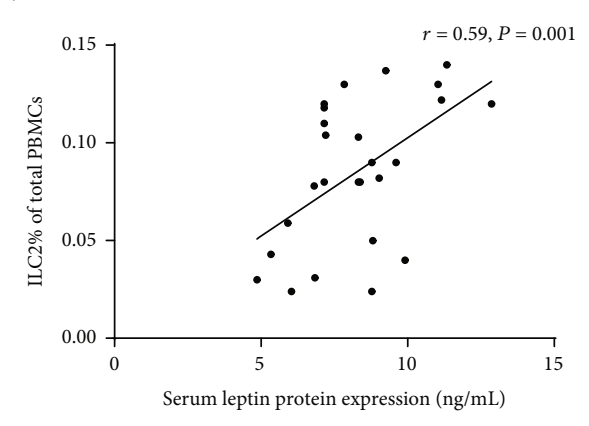

(e)

FIGURE 2: Flow cytometry confirmed elevated ILC2 frequency (a), IL- $4^{+}$ILC2 frequency (b), IL- $5^{+}$ILC2 frequency (c), and IL-13 ${ }^{+}$ILC2 frequency (d) in PBMCs from 26 AR compared with 20 controls. The correlation between serum levels of leptin and ILC2 frequency $\left(\operatorname{Lin}^{-} \mathrm{CD} 45^{+} \mathrm{CRTH} 2^{+} \mathrm{CD} 127^{+}\right.$) was shown in $(\mathrm{e}) .{ }^{*} \mathrm{P}<0.05$. Three independent tests were performed for every experiment.

culture supernatant of ILC2 isolated from blood of patients and leptin levels in serum was detected by ELISA kits (R\&D Systems, USA) as the protocol provided by the manufacturer. The sensitivity of the assays was as follows: IL-4, $0.22 \mathrm{pg} / \mathrm{mL}$; IL-5, $3.9 \mathrm{pg} / \mathrm{mL}$; IL-13, $125 \mathrm{pg} / \mathrm{mL}$; and leptin, $15.6 \mathrm{pg} / \mathrm{mL}$.
2.7. Animal Model. BALB/c mice were housed in the specific pathogen-free barrier facility with free access to food and water. On day 0 and day 7 , the mice were given $100 \mu \mathrm{L}$ of PBS containing $100 \mu \mathrm{g}$ of OVA $(1 \mu \mathrm{g} / \mu \mathrm{L})$ and $1.6 \mathrm{mg}$ $\mathrm{Al}(\mathrm{OH})_{3}$ intraperitoneally. The $10 \mu \mathrm{g}$ of OVA in $100 \mu \mathrm{L}$ of PBS with or without $10 \mu \mathrm{g} / \mathrm{mL}$ leptin, $10 \mu \mathrm{g} / \mathrm{mL}$ antileptin 


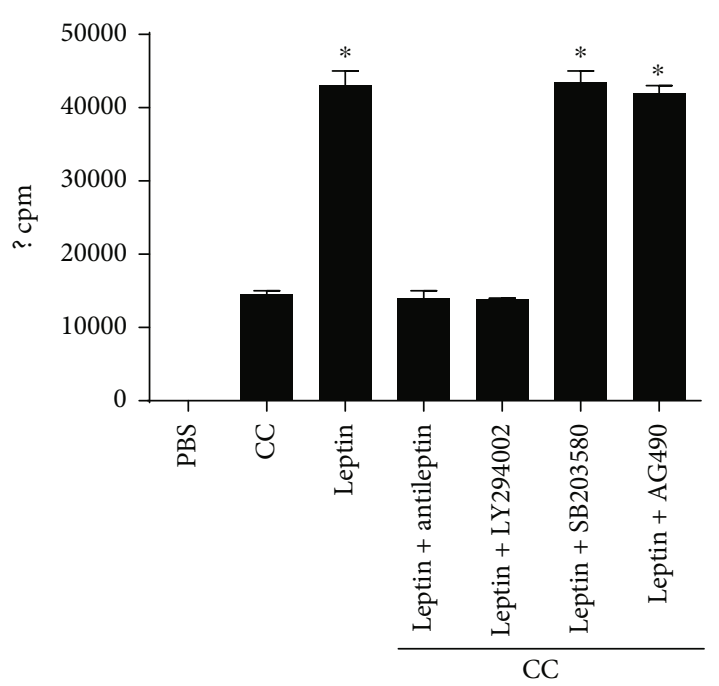

(a)

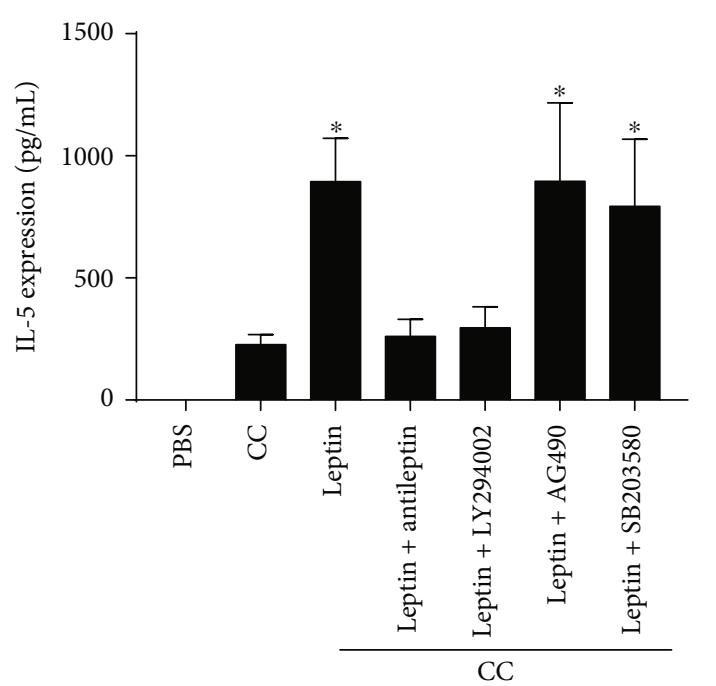

(c)

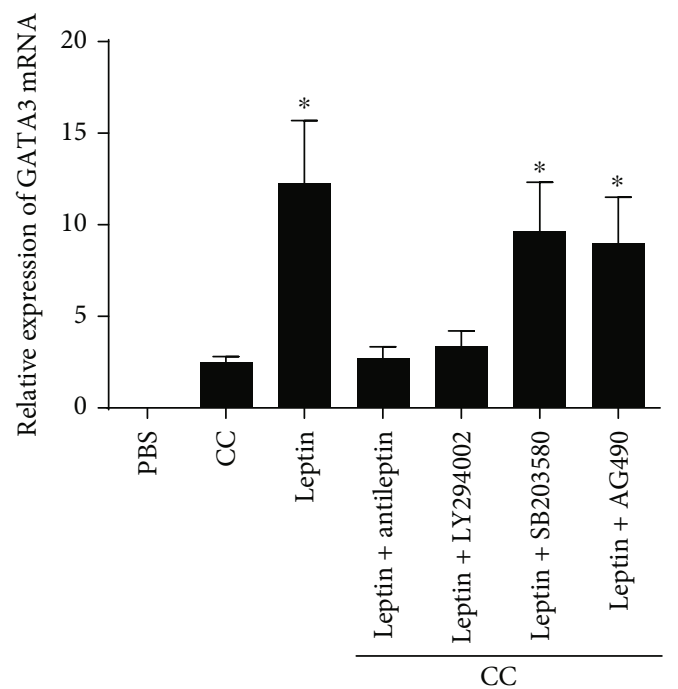

(e)

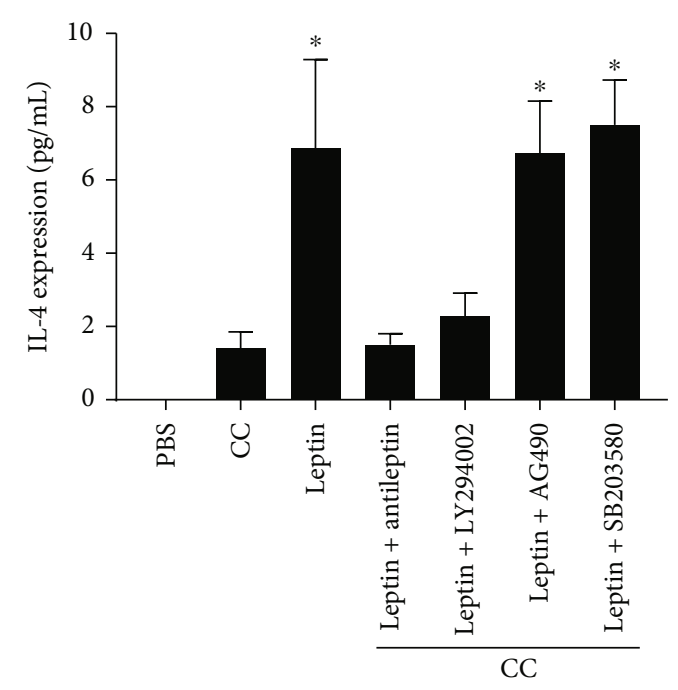

(b)

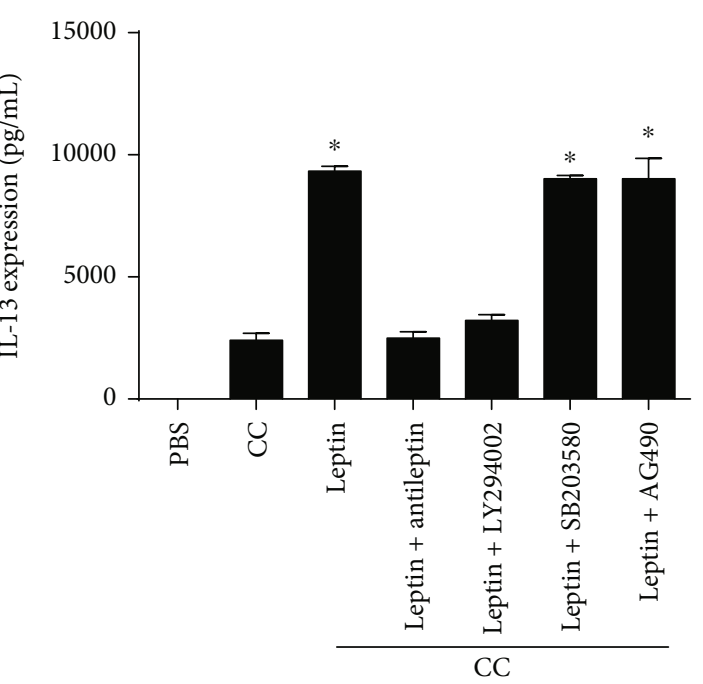

(d)

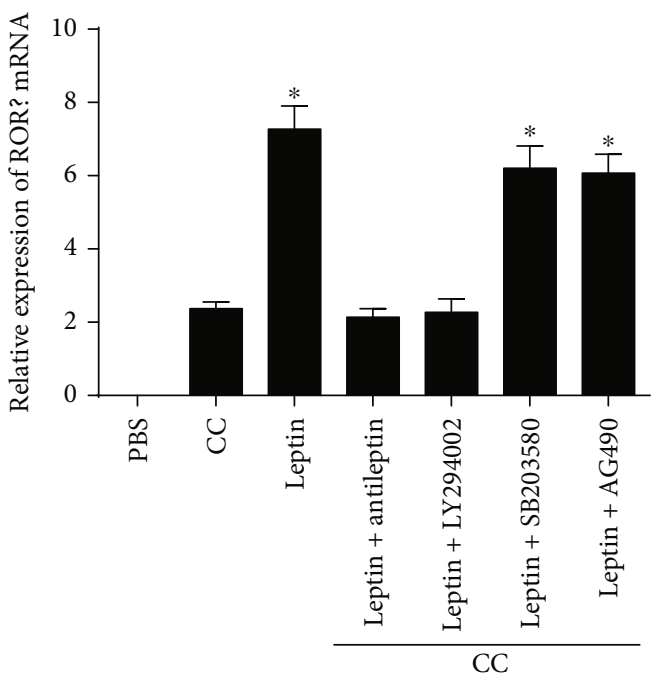

(f)

FIgure 3: Continued. 


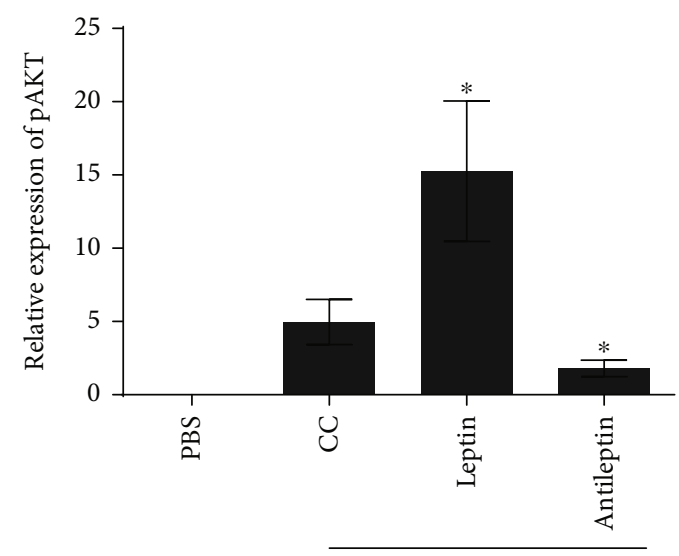

CC

(g)

FIGURE 3: Proliferation of ILC2 was assessed by using tritiated thymidine incorporation under different stimulation shown in (a). The protein expression of IL-4, IL-5, and IL-13 in the culture supernatant of ILC2 isolated from blood of patients tested by ELISA and mRNA expression of GATA3 and ROR $\alpha$ in ILC2 isolated from blood of 10 patients tested by RT-PCR after stimulated by leptin (b-f). The AKT phosphorylation was tested with or without leptin treatment in ILC2s (f). ${ }^{*} P<0.05$. LY294002, PI3K-specific inhibitor, AG490, JAK inhibitor, SB203580, and MAPK inhibitor. All the stimulators were $100 \mathrm{ng} / \mathrm{mL}$, and stimulation time was 3 days. Cytokine combinations (CC) of IL-25 (10 ng/mL), IL$33(10 \mathrm{ng} / \mathrm{mL})$, TSLP $(10 \mathrm{ng} / \mathrm{mL})$, and IL-2 $(50 \mathrm{ng} / \mathrm{mL})$ were added in all the cultures. Three independent tests were performed for every experiment.

(neutralization antibody), or $10 \mu \mathrm{g} / \mathrm{mL}$ LY294002 was given nasally on days 15,16 , and 19 with as previously described [16]. All cytokines and inhibitors were purchased from R\&D System. The mice were killed the day after the last nasal stimulation. The frequency of ILC2 cells in PBMCs was determined by flow cytometry.

2.8. Statistical Analysis. All data are showed as mean \pm SD. The Kruskal-Wallis $\mathrm{H}$ test or the nonparametric MannWhitney $U$ test was performed except additional note. Correlations between leptin and other parameters were assessed by the Spearman rank correlation analysis. A $P$ value of less than 0.05 was defined as statistically significant.

\section{Results}

3.1. The Serum Levels of Leptin and Its Correlation with ILC2 Frequency and Production of Type II Cytokines. The information of participants is listed in Table 1. The serum levels of leptin in the AR group were significantly higher compared with control $(9.8 \pm 2.3 \mathrm{ng} / \mathrm{mL} v s .3 .6 \pm 1.5 \mathrm{ng} / \mathrm{mL}, P<0.05)$. The frequency of ILC2, IL- $4^{+}$ILC2, IL- $5^{+}$ILC2, and IL-13 ${ }^{+}$ ILC2 was significantly elevated in AR patients compared with controls (Figures 1 and 2). The elevated serum leptin levels were significantly correlated with the frequency of ILC2 (Figure 2) and the frequency of $\mathrm{IL}^{+} 4^{+}$ILC2 $(r=0.46, P<0.05)$, IL-5 ${ }^{+}$ILC2 $(r=0.53, P<0.05)$, and IL$13^{+}$ILC2 $(r=0.71, P<0.05)$.

3.2. Expression of ILC2 Cell Transcription Factors and Type II Cytokine Stimulated by Leptin. After stimulated by leptin, the expression of GATA3 and $\operatorname{ROR} \alpha$ by ILC2 isolated from blood of patients was increased significantly. The type II cytokine production in the culture supernatant of ILC2 iso- lated from blood of patients was also upregulated after leptin stimulation. However, the above effect was inhibited when PI3K-AKT inhibitor or antileptin was added in the system (Figure 3).

3.3. Leptin Promotes ILC2 Responses in Mice. The serum leptin concentration, the level of OVA-specific IgE, the times of nasal rubbing and sneezing, and the frequency of ILC2 in PBMCs were significantly higher in AR mice than that of control mice, especially in the mice treated with leptin. Our results also showed that the antileptin attenuates allergic inflammation and the frequency of ILC2 (Figure 4).

\section{Discussion}

In this study, we explored the effects of leptin on ILC2 by cell and animal models. Our results confirmed that leptin can regulate the function of ILC2 by targeting the PI3K-AKT pathway.

Leptin is mainly secreted by the adipose tissue and also present in lymphoid organs $[10,11]$. Since the obese patients were excluded, we believed that lymphoid organs may be an important source of leptin. Moreover, the effect of leptin tolerance was also avoided by excluding the obese subjects. Therefore, further studies with obese AR patients were needed to clarify the role of leptin tolerance in innate immunity.

In adaptive immunity, leptin is found to induce Th1 response in both humans and mice, but its effect in proallergic Th2 response remains controversial. Recent studies proved that leptin promoted Th2 cell proliferation, survival, and cytokine production. Moreover, our previous study showed that leptin also involved Th17 immune responses by inducing $\operatorname{ROR} \gamma \mathrm{t}$ transcription in $\operatorname{AR}[6,7,17]$. Our results 


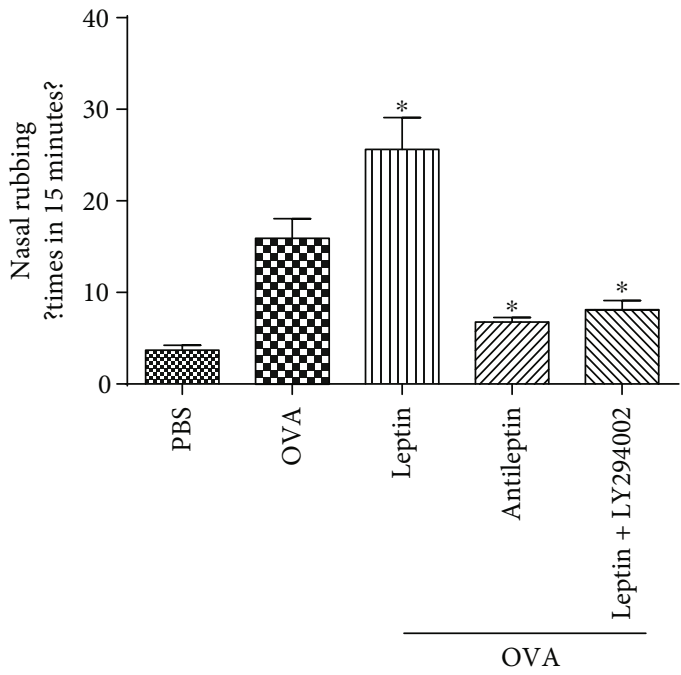

(a)

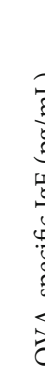

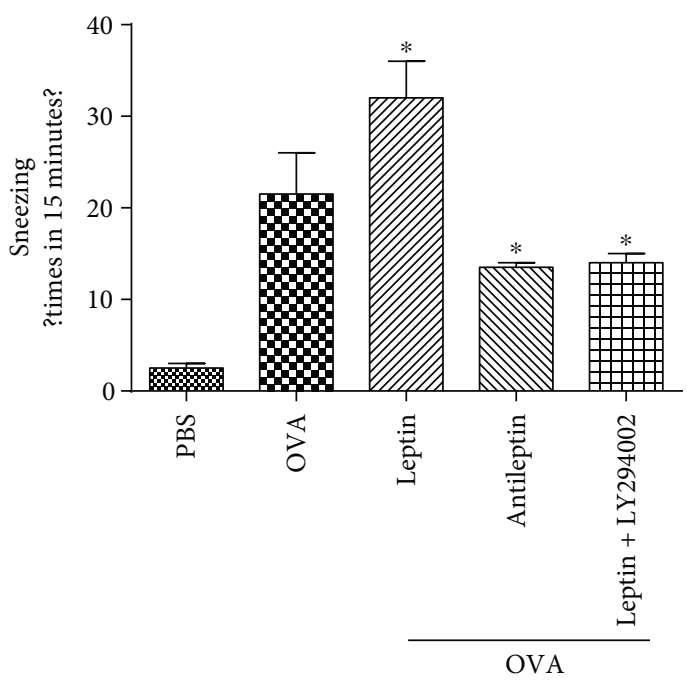

(b)

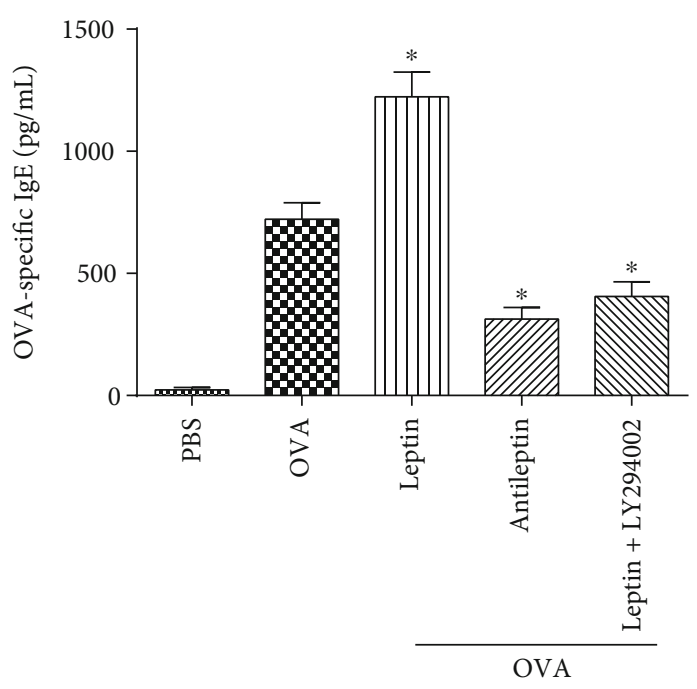

(c)

FIgURE 4: Continued. 

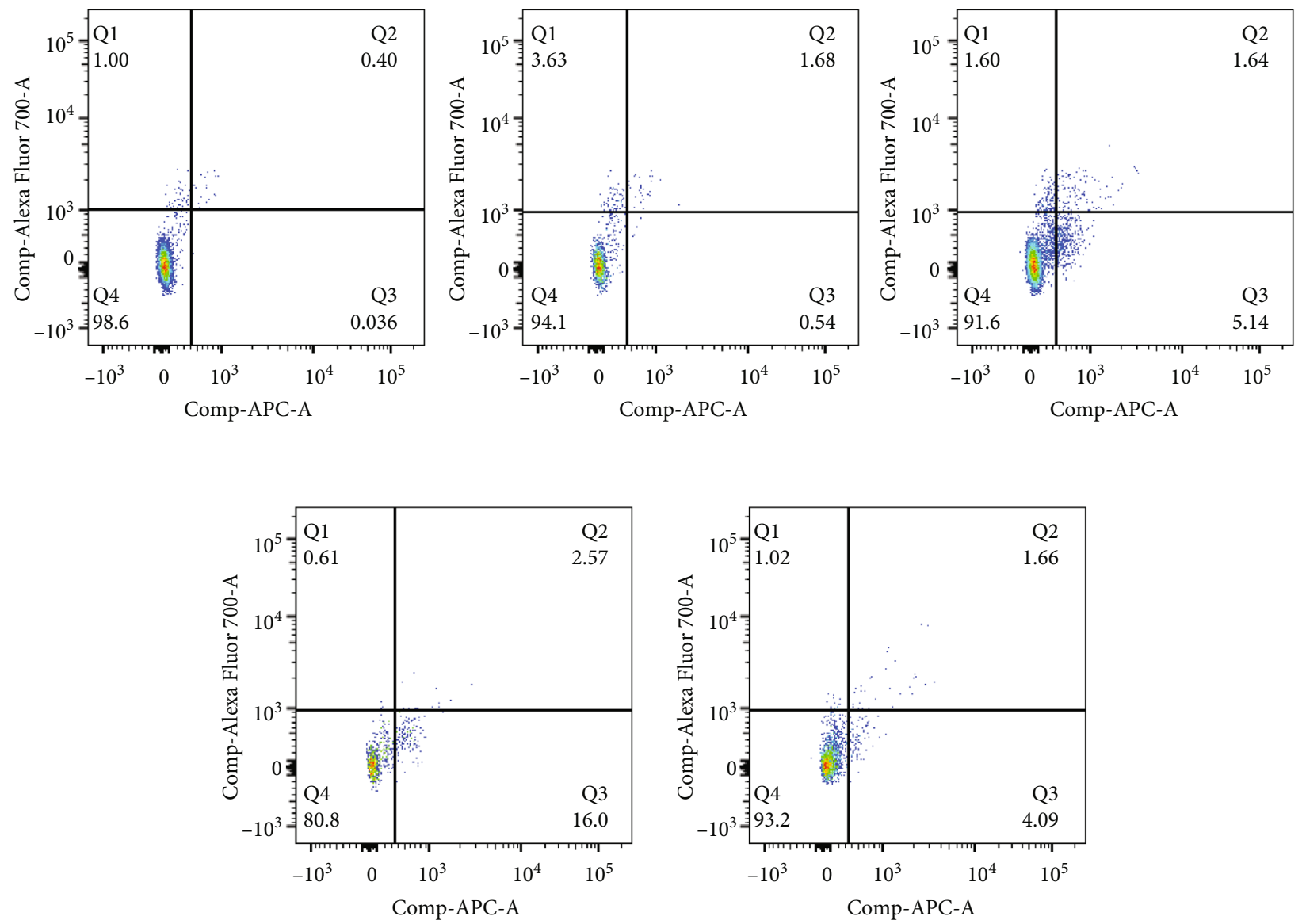

(d)

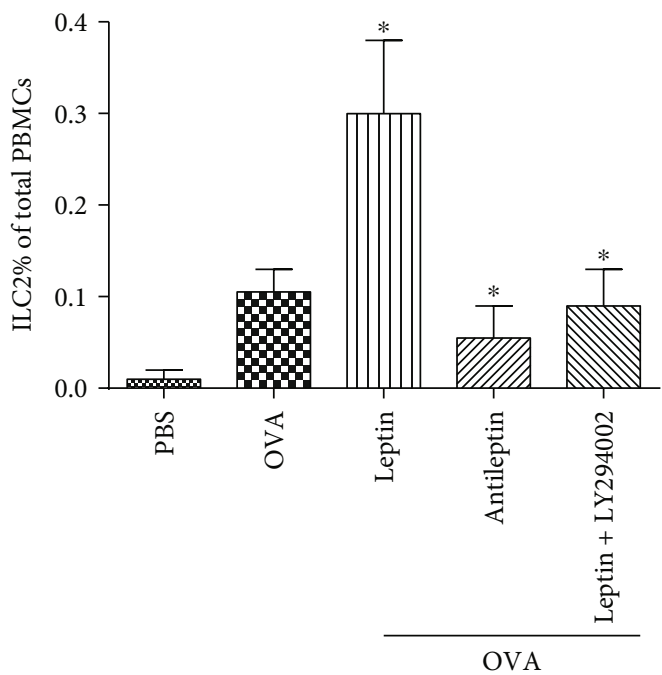

(e)

FIgURE 4: The times of nasal rubbing and sneezing ( $a, b)$, the serum OVA-specific IgE level determined by ELISA (c), and the frequency of ILC2 cells in PBMCs determined by flow cytometry in $30 \mathrm{AR}$ mice (five mice in every group) treated with leptin or antileptin (d, e). All the stimulators were $100 \mathrm{ng} / \mathrm{mL}$. All the frequencies of ILC2s were converted into proportions in PBMCs (per mL of blood). ${ }^{*} P<0.05$. LY294002, PI3K-specific inhibitor. Three independent tests were performed for every experiment.

also suggested that local treatment of leptin promotes systemic IgE production, which may be attributed to the effect of elevated local expression of IL-4 on bone marrow through blood circulation. Besides, the proliferation and cytokine production by Th2 cell promoted by leptin can also induce the expression of IL-4, which contribute to IgE production in both humans and mice. However, the role of leptin in innate immunity was not characterized well. 
In the present study, we found that the serum levels of leptin were significantly higher in AR patients compared with controls and correlated with the level of type II cytokines. We also demonstrated that leptin, an adipokine elevated in obese individuals, promoted the function of ILC2, proved by upregulated expression of GATA3 and ROR $\alpha$ and type II cytokine production. Consistently, Zheng et al.'s study also showed that leptin promoted proliferation and survival of Th2 cells and ILC2 and production of type 2 cytokines, which together contribute to allergic asthma [18].

Leptin acts to regulate immune cell function by multiple signaling pathways, such as JAK2-STAT3, MAPK, and PI3KAKT $[10,11]$. For example, the leptin-STAT3 pathway has been proved to mediate cell survival in hippocampal neurons [19]. Moreover, leptin exerted an antiapoptotic effect by activating ERK1/2 and AKT-mTOR pathways in Th1/Th17 cells [20]. Interestingly, the antiapoptotic effects of leptin are dependent on MAPK activation, rather than the PI3K pathway in human Jurkat T cells [21]. Our present study showed that leptin mediated the effect of ILC2 through the PI3KAKT pathway in AR. Our in vivo study also suggested that leptin promoted ILC2 inflammation through the PI3KAKT pathway, which confirmed the in vitro results.

There are also some limitations in our study. First of all, the sample size is small and further studies with large sample size are needed. Secondly, the obese subject in our study was excluded to eliminate the effect of obese state on the expression of leptin. Therefore, the study of leptin on the regulation of ILC2 in obese AR patients was needed. Thirdly, further studies with Rag-deficient mice lack of lymphocytes should be used to confirm the effects of leptin in ILC2s. Fourthly, systemic treatment of leptin in mouse model should be studied to explore the systemic effect of leptin on the regulation of ILC2.

\section{Conclusions}

In summary, our findings suggested leptin-mediated regulation of the differentiation and function of ILC2 cells through leptin and PI3K-AKT signaling pathway, providing a new potential treatment target.

\section{Abbreviations}

AR: $\quad$ Allergic rhinitis

ILC2: $\quad$ Type II innate lymphoid cells

ELISA: Enzyme-linked immunosorbent assay

qRT-PCR: Quantitative real-time PCR.

\section{Data Availability}

The datasets used and/or analyzed during the current study are available from the corresponding author on reasonable request.

\section{Ethical Approval}

The study protocols were approved by local ethics committee boards.

\section{Consent}

Written informed consent was obtained.

\section{Conflicts of Interest}

The authors declare that they have no relevant conflicts of interest.

\section{Authors' Contributions}

Dr. Wenlong Liu and Renzhong Luo conceptualized and designed the study, drafted the initial manuscript, and approved the final manuscript as submitted. Dr. Qingxiang Zeng, Xi Luo, and Yiquan Tang collected the sample; performed the experiment, data collection, and statistics; reviewed and revised the manuscript; and approved the final manuscript as submitted. Qingxiang Zeng, Xi Luo, and Yiquan Tang contributed equally to this study.

\section{Acknowledgments}

This study was supported by grants from the National Natural Science Grant of China (No. 81600785, No. 81700892, and No. 81970861), the Pearl River S\&T Nova Program of Guangzhou (No. 201710010085), and the Key Clinical Specialty of Guangzhou Women and Children's Medical Center, Grant of the Institute of Pediatrics of Guangzhou Women and Children's Medical Center (YIP-2016-022, Pre-NSFC2018-005).

\section{References}

[1] J. L. Brożek, J. Bousquet, C. E. Baena-Cagnani et al., "Allergic Rhinitis and its Impact on Asthma (ARIA) guidelines: 2010 revision," The Journal of Allergy and Clinical Immunology, vol. 126, no. 3, pp. 466-476, 2010.

[2] Y. Wang and T. Lobstein, "Worldwide trends in childhood overweight and obesity," International Journal of Pediatric Obesity, vol. 1, no. 1, pp. 11-25, 2006.

[3] C. Papoutsakis, K. N. Priftis, M. Drakouli et al., "Childhood overweight/obesity and asthma: is there a link? A systematic review of recent epidemiologic evidence," Journal of the Academy of Nutrition and Dietetics, vol. 113, no. 1, pp. 77-105, 2013.

[4] A. C. Goldberg, F. Goldberg-Eliaschewitz, M. C. Sogayar, J. Genre, and L. V. Rizzo, "Leptin and the immune Response," Annals of the New York Academy of Sciences, vol. 1153, no. 1, pp. 184-192, 2009.

[5] G. M. Lord, G. Matarese, J. K. Howard, R. J. Baker, S. R. Bloom, and R. I. Lechler, "Leptin modulates the T-cell immune response and reverses starvation-induced immunosuppression," Nature, vol. 394, no. 6696, pp. 897-901, 1998.

[6] D. M. Youssef, R. M. Elbehidy, D. M. Shokry, and E. M. Elbehidy, "The influence of leptin on Th1/Th2 balance in obese children with asthma," Jornal Brasileiro de Pneumologia, vol. 39, no. 5, pp. 562-568, 2013.

[7] A. Batra, B. Okur, R. Glauben et al., "Leptin: a critical regulator of $\mathrm{CD}^{+}{ }^{+} \mathrm{T}$-cell polarization in vitro and in vivo," Endocrinology, vol. 151, no. 1, pp. 56-62, 2010. 
[8] K. C. Hsueh, Y. J. Lin, H. C. Lin, and C. Y. Lin, "Serum leptin and adiponectin levels correlate with severity of allergic rhinitis," Pediatric Allergy and Immunology, vol. 21, 1 Part 2, pp. e155-e159, 2010.

[9] G. Ciprandi, M. De Amici, M. A. Tosca, and G. Marseglia, "Serum leptin levels depend on allergen exposure in patients with seasonal allergic rhinitis," Immunological Investigations, vol. 38, no. 8, pp. 681-689, 2009.

[10] G. Matarese, S. Moschos, and C. S. Mantzoros, "Leptin in immunology," Journal of Immunology, vol. 174, no. 6, pp. 3137-3142, 2005.

[11] G. Fantuzzi and R. Faggioni, "Leptin in the regulation of immunity, inflammation, and hematopoiesis," Journal of Leukocyte Biology, vol. 68, no. 4, pp. 437-446, 2000.

[12] M. Flach and A. Diefenbach, "Adipose tissue: ILC2 crank up the heat," Cell Metabolism, vol. 21, no. 2, pp. 152-153, 2015.

[13] A. B. Molofsky, J. C. Nussbaum, H.-E. Liang et al., "Innate lymphoid type 2 cells sustain visceral adipose tissue eosinophils and alternatively activated macrophages," The Journal of Experimental Medicine, vol. 210, no. 3, pp. 535-549, 2013.

[14] A. B. Molofsky, F. Van Gool, H.-E. Liang et al., "Interleukin-33 and interferon- $\gamma$ counter-regulate group 2 innate lymphoid cell activation during immune perturbation," Immunity, vol. 43, no. 1, pp. 161-174, 2015.

[15] X. Ding, Y. Luo, X. Zhang et al., "IL-33-driven ILC2/eosinophil axis in fat is induced by sympathetic tone and suppressed by obesity," The Journal of Endocrinology, vol. 231, no. 1, pp. 35-48, 2016.

[16] M. Nakaya, M. Dohi, K. Okunishi et al., "Prolonged allergen challenge in murine nasal allergic rhinitis: nasal airway remodeling and adaptation of nasal airway responsiveness," Laryngoscope, vol. 117, no. 5, pp. 881-885, 2007.

[17] W. Liu, Q. Zeng, L. Zhou, Y. Li, Y. Chen, and R. Luo, "Lepti$\mathrm{n}$ /osteopontin axis contributes to enhanced $\mathrm{T}$ helper 17 type responses in allergic rhinitis," Pediatric Allergy and Immunology, vol. 29, no. 6, pp. 622-629, 2018.

[18] H. Zheng, X. Zhang, E. F. Castillo, Y. Luo, M. Liu, and X. O. Yang, "Leptin enhances TH2 and ILC2 responses in allergic airway disease," The Journal of Biological Chemistry, vol. 291, no. 42, pp. 22043-22052, 2016.

[19] Z. Guo, H. Jiang, X. Xu, W. Duan, and M. P. Mattson, "Leptinmediated cell survival signaling in hippocampal neurons mediated by JAK STAT3 and mitochondrial stabilization," The Journal of Biological Chemistry, vol. 283, no. 3, pp. 1754-1763, 2008.

[20] B. Mattioli, L. Giordani, M. G. Quaranta, and M. Viora, "Leptin exerts an anti-apoptotic effect on human dendritic cells via the PI3K-Akt signaling pathway," FEBS Letters, vol. 583, no. 7, pp. 1102-1106, 2009.

[21] P. Fernandez-Riejos, R. Goberna, and V. Sanchez-Margalet, "Leptin promotes cell survival and activates Jurkat T lymphocytes by stimulation of mitogen-activated protein kinase," Clinical and Experimental Immunology, vol. 151, no. 3, pp. 505-518, 2008. 